\title{
SUR LE PROBLĖME DE WATSON DANS LA THÉORIE DES SÉRIES ASYMPTOTIQUES ET SOLUTION D'UN PROBLĖME DE CARLEMAN DE LA THÉORIE DES FONCTIONS QUASI- ANALYTIQUES.
}

\author{
PAR
}

R، SAN JUAN

à MADR1D.

Dans cet article, nous résolvons un problème proposé par $\mathbf{M}$. Carleman dans son ouvrage "Les fonctions quasi-analytiques" (Paris 1926, pag. 77) ainsi que le problème qui lui correspond dans la théorie des séries asymptotiques, problème qui se présente tout naturellement quand on aborde celui de l'unicité de la somme dans la théorie des séries divergentes.

M. Carleman, avec l'esprit de pénétration qui le caractérise, avait remarqué déjà que ce problème ne peut pas être résolu au moyen des procédés habituels de la théorie des fonctions quasi-analytiques et qui consiste à réduire l'identité ou la diversité de deux fonctions, à l'annulation ou non de leur différence. Pour résoudre ce problème il faut une »méthode de décomposition»: C'est cette méthode que nous exposons dans les paragraphes I et II pour une génératrice de Stieltjes et de Laplace respectivement ${ }^{1}$ mais qui pourrait être utilisé pour un algorithme quelconque avec génératrice, c'est-à-dire, intégrale ou série de la forme

$$
\int_{0}^{\infty} \alpha(t) \Phi(t, z) d t \quad \text { ou } \sum_{t=0}^{\infty} \alpha_{t} \Phi_{t}(z)
$$

1 Un résumé fut présenté dans le Congrès d'Oslo (R. SAs JUAN. "Sur le probleme de Watson etc. Extrait des Atti du Congrès international des Mathématiciens. 1936, II pag. 94. 
les fonctions $\boldsymbol{\Phi}(t, z)$ ou $\boldsymbol{\Phi}_{t}(z)$ étant de types préfixés: fonctions entières, polynômes ordinaires on trigonométriques ${ }^{1}$, fractions simples, etc.

Le procédé indiqué, qui permet d'obtenir des fonctions différentes ayant des dérivées égales en un point sans y ajouter une fonction, de dérivée zéro, comme on le fait d'habitude, nous paraît susceptible d'applications dans la théorie des fonctions quasi-analytiques. Il est en effet d'une portée plus grande que le premier auquel nous faisons allusion et dans lequel l'une des fonctions était $f(x) \equiv 0$; lorsqu'il s'âgit, par exemple, de démontrer la nécessité des conditions de quasianalyticité, de caractériser les successions, qui rendent quasi-analytique la famille de fonctions holomorphes définies par des séries de fractions simples

$$
\sum_{n=0}^{\infty} \frac{A_{n}}{z-\boldsymbol{\alpha}_{n}}{ }^{2} \text { ete, ete. }
$$

I. - On sait qu'une même série peut être le développement asymptotique d'un nombre infini de fonctions, et c'est précisément dans cette indétermination que réside la cause de ce que l'on n'adopte pas la fonction asymptotique comme valeur de la série dans la théorie de séries divergentes, dans laquelle le problème de l'unicité est, comme on l'a déjà dit $^{3}$ le point le plus délicat de toute la théorie. Mais la définition de Poincaré peut être convenablement modifiée, de façon que la fonction reste univoquement déterminée par la série, et que les propriétés restantes des développements asymptotiques continuent à subsister. M. Watson a été le premier à aborder ce problème. C'est dans son intéressant mémoire "A theory of asymptotic series" (Trans. of the Royal Society of London, série A vol. 211, pag. 279-313) qu'il démontre l'unicité quand les bornes de Poincaré

$$
A_{n} \geq\left|\frac{F(z)-\sum_{v=0}^{n-1} a_{v} z^{v}}{z^{n}}\right|
$$

${ }^{1}$ Par exemple, l'application aux séries de polyoômes de Tchebychef $\sum_{n=0}^{\infty} a_{n} \cos (n$ arc. cos $x)$ a été faite par M. Mandelbrojt qui a connu cette même exposition détaillée l'année 1938 par l'entremise de M. Gorny. M. Mandelbrojt a généralisé notre résultat et a démontré un joli théorème sur les fonctions indéfiniment dérivables, qui permet de répondre d'une manière plus large à la demande de M. Carleman (Acta mathematica t. 72, p. I6).

${ }^{2}$ Carleman, loc. cit. pag. 97 ou bien A. Densoy. Introduction à la théorie des fonctions de variable réelle. Actualités scientifiques et industrielles. Paris 1937, pag. 41.

'Borel. Mémoire sur les séries divergentes. Annales scientifiques de l'École Normale Supérieure (I899). KoGBetuins. Sommation de séries et intégrales divergentes etc. Mémorial des sciences mathématiques T. LX (193I). 
Sur le problème de Watson dans la théorie des séries asymptotiques. $\quad 249$

dans un secteur angulaire de sommet o, croissent moins rapidement que $k^{n} \cdot\lfloor(\mathrm{I}-\varepsilon) n$ c'est-à-dire:

$$
A_{n}<k^{n} \cdot \underline{(\mathrm{I}-\varepsilon) n}
$$

$\varepsilon$ étant $>0$ et $k$ une constante convenante.

M. Nevanlinna, dans son travail »Zur Theorie der asymptotischen Potenzreihen» (Akademische Abhandlungen, Helsingfors, 1918) généralisa immédiatement la solution en supprimant $\varepsilon$, et plusieurs années plus tard, M. Carleman, dans son précieux ourrage "Les fonctions quasi-analytiques», Paris, I926, pose le problème général d'obtenir les conditions non seulement suffisantes mais en même temps nécessaires, que doivent remplir les bornes $A_{n}$ relatives au cercle $|\mathrm{I}-z|<\mathrm{I}$ pour que les limitations de Poincaré entraînent la détermination de $F(z)$, en donnant comme solution la divergence de l'intégrale

$$
\int_{1}^{\infty} \log \sum_{v=0}^{\infty} \frac{x^{2 v}}{A_{v}^{2}} \cdot \frac{d x}{x^{2}}=\infty
$$

ou ce qui revient au même, que la plus petit majorante non croissante de $\sum \frac{I}{v}$ soit divergente, c'est-à-dire:

$\angle \sqrt{A_{v}}$

$$
\sum_{\nu=0}^{\infty} \frac{1}{a_{v}^{\prime}}=\infty
$$

$\alpha_{v}^{\prime}$ étant la borne inférieure de l'ensemble $\sqrt[v]{A_{v}}, \sqrt[v+1]{\sqrt{A_{v+1}}}, \sqrt[v+\dot{2}]{\sqrt{A_{v+2}}}, \ldots$

Comme il arrive dans tout problème de ceux que Borel a appelé d'interpolation linéaire en sens large ${ }^{1}$, la fonction ne reste pas déterminée par la série asymptotique seulement, sinon par celle ci et, en outre, par une condition complémentaire que MM. Watson, Nevanlinna et Carleman ont imposée aux bornes de Poincaré:

$$
A_{n}>\left|\frac{F(z)-\sum_{v=0}^{n-1} a_{v} z^{v}}{z^{n}}\right| .
$$

Mais tandis que les théorèmes de MM. Watson et Nevanlinna donnent une seule condition d'unicité:

$$
A_{n}<k^{n} \underline{\underline{(\mathrm{I}-\varepsilon) n}} \text { ou bien } A_{n}<k^{n}\lfloor n
$$

${ }^{1}$ BoREL. Leçons sur les séries divergentes. pag. 35.

32-41841. Acta mathematica. 75. Imprimó le 15 juin 1943. 
le théorème de $M$. Carleman en donne un nombre infini, car il y a un nombre infini de successions différentes $A_{n}$ qui rendent l'intégrale

$$
\int_{1}^{\infty} \log \sum_{v=0}^{\infty} \frac{x^{2 v}}{A_{\nu}^{2}} \cdot \frac{d x}{x^{2}}=\infty
$$

et il se présente le problème de savoir si deux fonctions qui remplissent la condition de $\mathbf{M}$. Carleman avec un même développement asymptotique, mais avec des bornes différentes, coïncident, c'est-à-dire, si les conditions

$$
\begin{gathered}
\left|\frac{F(z)-\sum_{v=0}^{n-1} a_{v} z^{v}}{z^{n}}\right|<A_{n}\left|\frac{F_{1}(z)-\sum_{v=0}^{n-1} a_{v} z^{v}}{z^{n}}\right|<A_{n}^{\prime} \text { pour }|\mathrm{I}-z|<\mathrm{I} \\
\quad \int_{1}^{\infty} \log \sum_{v=0}^{\infty} \frac{x^{2 v}}{A_{v}^{2}} \cdot \frac{d x}{x^{2}}=\infty \\
\int_{1}^{\infty} \log \sum_{v=0}^{\infty} \frac{x^{2 v}}{A_{v}^{\prime 2}} \cdot \frac{d x}{x^{2}}=\infty
\end{gathered}
$$

entraînent $F(z) \equiv F_{1}(z)$.

Pour repondre à cette question nous allons définir une suite de nombres entiers $t_{n}$ et $\bar{t}_{n}$ tels que

$$
0<t_{1}<\bar{t}_{1}<t_{2} \cdots<\bar{t}_{n-1}<t_{n}<\bar{t}_{n} \cdots, \quad t_{n} \rightarrow \infty
$$

Prenons d'abord un $t_{1}$ suffisamment grand pour que

$$
\int_{t_{1}}^{\infty} e^{-\sqrt[4]{t}^{\bar{t}}} t^{i} d t<\mathrm{I} \quad \text { pour } \quad i=\mathrm{I}=v_{1}
$$

et ensuite un $t_{1}$ de telle manière que

$$
\int_{\bar{t}_{1}}^{\infty} e^{-\sqrt[4]{t}} t^{i} d t<t_{1}^{i+1} \quad \text { pour } \quad i=\mathrm{I}, 2, \ldots\left(2 t_{1}\right)^{2}=\bar{v}_{1}
$$

Cela étant, pour $n>\mathrm{I}$, les nombres $t_{n}, \bar{t}_{n}$ sont déterminés par les conditions 
Sur le problème de Watson dans la théorie des séries asymptotiques.

$$
\begin{aligned}
& \int_{t_{n}}^{\infty} e^{-\sqrt[4]{t}} t^{i} d t<\left(\bar{t}_{n-1}\right)^{i+1} \quad \text { pour } \quad i=v_{n-1}+\mathrm{I}, v_{n-1}+2 \ldots v_{n-1}+\left(2 \bar{t}_{n-1}\right)^{2}=i_{n} \\
& \int_{\bar{t}_{n}}^{\infty} e^{-\sqrt[4]{t}} t^{i} d t<\left(t_{n}\right)^{i+1} \quad \text { pour } \quad i=\bar{v}_{n-1}+\mathrm{I}, \ldots, \bar{v}_{n-1}+\left(2 t_{n-1}\right)^{2}=\bar{v}_{n} .
\end{aligned}
$$

La fonction $\alpha(t)$ est par définition égale à o dans $\left(o, t_{1}\right)$ et dans tout intervalle $\left(\bar{t}_{n-1}, t_{n}\right)$; dans les intervalles restants $\alpha(t)=e^{-\bar{t}^{t}}$. La fonction $\bar{\alpha}(t)$ est égale à o dans les intervalles $\left(t_{n}, \bar{t}_{n}\right)$; dans les intervalles restants $\bar{\alpha}(t)=e^{-\sqrt[4]{t}^{\bar{t}}}$. Donc $\alpha(t)+\bar{\alpha}(t)=e^{-\sqrt[4]{\bar{t}}}$ pour $t \geq 0$. Posons

$$
\mu_{i}=\int_{0}^{\infty} \alpha(t) t^{i} d t, \quad \bar{\mu}_{i}=\int_{0}^{\infty} \bar{\alpha}(t) t^{i} d t .
$$

On a

$$
\begin{gathered}
\mu_{1}=\int_{t_{1}}^{\infty} e^{-\sqrt[4]{t}} t d t<\mathrm{I} \\
\mu_{i}<\int_{0}^{\bar{t}_{n}} \alpha(t) t^{i} d t+\int_{t_{n+1}}^{\infty} e^{-\bar{V}^{t}} d t<2\left(\bar{t}_{n}\right)^{i+1} \quad \text { pour } \quad i=v_{n}+\mathrm{I}, \ldots v_{n}+\left(2 \bar{t}_{n}\right)^{2}
\end{gathered}
$$

done

et

$$
\sum_{i=v_{n}+1}^{v_{n+1}} \frac{\mathrm{I}}{i} \sqrt{\sqrt{\mu_{i}}}>\frac{\left(2 \bar{t}_{n}\right)^{2}}{\left(2 \bar{t}_{n}\right)^{\frac{1}{v_{n}+1}} \cdot \bar{t}_{n}}>\mathrm{I}
$$

$$
\sum_{I}^{\infty} \frac{I}{\sqrt[i]{\mu_{i}}}=\infty
$$

De la même manière on trouvera

$$
\bar{\mu}_{i}<\int_{0}^{t_{n}} \bar{\alpha}(t) t^{i} d t+\int_{\bar{t}_{n}}^{\infty} e^{-\sqrt[4]{t}^{\bar{t}}} t^{i} d t<2\left(t_{n}\right)^{i+1} \quad \text { pour } \quad i=\bar{v}_{n-1}+\mathrm{I}, \ldots \bar{v}_{n}
$$

et

$$
\sum_{i=1}^{\infty} \frac{1}{\sqrt{\bar{\mu}_{i}}}=\infty
$$


Mais

$$
\begin{gathered}
\int_{0}^{\infty} \alpha(t) \sin (\sqrt[4]{t}) t^{n} d t+\int_{0}^{\infty} \bar{\alpha}(t) \sin \left(\sqrt{ }^{4} \bar{t}\right) t^{n} d t=\int_{0}^{\infty} e^{-\sqrt[4]{t}} \sin (\sqrt[4]{t}) t^{n} d t=0 \\
\quad \operatorname{pour} n=\mathrm{I}, 2,3, \ldots ;
\end{gathered}
$$

d'où il s'ensuit que les fonctions:

$$
A(t)=\alpha(t) \sin (\sqrt[4]{t}) \quad A_{1}(t)=-\bar{\alpha}(t) \sin (\sqrt[4]{t})
$$

ont des moments égaux:

$$
c_{n}=\int_{0}^{\infty} A(t) t^{n} d t=\int_{0}^{\infty} A_{1}(t) t^{n} d t
$$

et par conséquent les fonctions:

$$
F(z)=\int_{0}^{\infty} \frac{A(t) d t}{t+z} \quad F_{1}(z)=\int_{0}^{\infty} \frac{A_{1}(t) d t}{t+z}
$$

qui sont différentes, puisque

$$
F(z)-F_{1}(z)=\int_{0}^{\infty} \frac{e^{-\sqrt{4}^{t}} \sin \sqrt{t} t}{t+z}
$$

ont le même développement asymptotique $\sum(-\mathrm{I})^{n} \frac{c_{n}}{z^{n+1}}$ avec des bornes $A_{n}=\mu_{n}$ et $A_{n}^{\prime}=\mu_{n}^{\prime}$ qui remplissent toutes deux la condition de M. Denjoy et, par suite, celle de Carleman.

$I l$ existe donc des séries qui sont le développement asymptotique de fonctions distinctes, avec des bornes différentes qui remplissent la condition de $\boldsymbol{M}$. Carleman ${ }^{\mathbf{1}}$.

1 Une condition suffisante pour la coïncidence de toutes les fonctions qui admettent un même développement asymptotique $\Sigma a_{n} z^{n}$ avec bornes de Carleman, est $q u$ 'il existe une fonction dont les bornes remplissent la condition:

$$
\varlimsup_{n \rightarrow \infty} \sqrt{\frac{A_{n}}{\left|a_{n}\right|}}<\infty .
$$

Nous n'avons pas démontré que cette condition est suffisante, mais on voit inmédiatement qu'elle est vérifiée pour toutes les séries que nous connaissons (G. N. Watson, A theory of asymptotic series, Phil. Trans. Royal Soc. London. Ser. A, vol. 211, p. 3I2; The caracteristic of asymptotic series. Some analitic associated with the $G$-function. The Quarterly Jour. of Pure and Applied Mathematics. $n^{\circ} 169,1911 .-n^{\circ} 174,1913$ resp.). 
Sur le problème de Watson dans la théorie des séries asymptotiques.

La condition nécessaire et suffisante pour que toutes les fonctions qui ont un même développement asymptotique avec des bornes différentes qui remplissent la condition de Carleman cö̈ncident, est que ce développement admette une fonction d'approximation asymptotique optime, c'est-à-dire, dont les plus petites bornes en soient ordonnément moindres que les homologues $A_{n}^{\prime}$ de toute autre fonction quelconque sauf un facteur exponentiel: $A_{n}<A_{n}^{\prime} \cdot K^{n 1}$.

Comme fonction d'approximation asymptotique optime, nous arons le prolongement analytique ordinaire et aussi les fonctions qui remplissent la condition de Watson-Nevanlinna, car $A_{n}+n$ ! remplit la condition de Carleman si $A_{n}$ la réalise. Les fonctions que Stieltjes assignait à ses séries alternées sont aussi d'approximation optime, parce qu'alors les bornes sont les coefficients de la série même. On démontre d'une manière analogue que la fonction exprimée par la série de Stirling et les intégrales de Fresnel qui se développent dans les séries de Cauchy, etc. sont d'approximation optime. C'est donc la fonction d'approximation optime qui, à l'exclusion de toutes les autres, doit être considérée comme définie par la série à rayon nul, d'après la phrase de Le Roy.

On voit facilement que toute valeur assignée à une série de puissances est d'approximation optime si elle satisfait aux lois formelles d'unicité, d'holomorphie, distributive et de suppression du terme initial. Cette notion résout donc le problème d'unicité dans la théorie des séries divergentes de la manière la plus ample possible, compatible arec ces lois formelles du calcul; et pour certaines algorithmes, comme celui de M. Borel, qui ne permettent pas la suppression du terme initial cette conclusion subsiste lorsque la somme est asymptotiquement approchée par la série, ce qui est l'unique cas d'intérêt analytique.

II. - Au moyen de la transformation de Laplace, chaque problème de moments se convertit en un autre de fonctions quasi-analy tiques réelles et celui-ci, à son tour, en un autre de séries asymptotiques. Ainsi donc, au moyen des fonctions :

$$
f(x)=\int_{0}^{\infty} A(t) e^{-t x} d t \quad f_{1}(x)=\int_{0}^{\infty} A_{1}(t) e^{-t x} d t
$$

${ }^{1}$ Quelques propriétés de cette notion, que nous avons introduite dans notre Mémoire * Sommation de séries divergentes et approximation asymptotique optime” présenté a l'Académie des Sciences de Madrid en 1934 peuvent être vues dans notre article "Dérivation et intégration de séries asymptotiques* (Unión Matemática Argentina, 1938).

${ }^{2}$ LE Roy, Sur les séries divergentes et sur les fonctions définies par nn développement de Taylor. Annales de la Faculté des Sciences de Tonleuse (Igoo) page 312. 
on trouve la réponse négative à la demande de Carleman (page 77 de son livre mentioné plus hant) sur la coïncidence de deux fonctions réelles qui ont des dérivées égales en un point:

$$
f^{v}(0)=f_{1}^{v}(0) \quad(y=0,1,2,3, \ldots)
$$

et remplissent la condition de Denjoy, e'est-à-dire:

$$
\sum_{v=1}^{\infty} \frac{\mathrm{I}}{\sqrt[v]{\mu_{v}}}=\infty \text { et } \sum_{v=1}^{\infty} \frac{\mathrm{I}}{\sqrt{\mu_{v}^{\prime}}}=\infty
$$

$\mu_{v}$ et $\mu_{v}^{\prime}$ étant les maximums de $\left|f^{v}(x)\right|$ et $\left|f_{1}^{v}(x)\right|$ respectivement dans l'intervalle (o, $\left.a\right)$.

Il s'ensuit qu'il $\mathrm{y}$ a des classes quasi-analytiques, comme celles $C_{\mu}$ et $C_{\mu^{\prime}}$ définies par les limitations

$$
\begin{aligned}
& \left|f^{n}(x)\right|<K^{n} \cdot \mu_{n} \\
& \left|f_{1}^{n}(x)\right|<K_{1}^{n} \cdot \mu_{n}^{\prime}
\end{aligned}
$$

dont l'ensemble agrégé $C_{\mu}+C_{\mu^{\prime}}$ n'est pas quasi-analytique $\Delta$, avec la notation de M. Mandelbrojt ${ }^{1}$ et par conséquent qu'il n'y a pas de classe quasi-analytique qui comprenne toutes les autres, parce que, si elle contenait $C_{\mu}$ et $C_{\mu}$, elle contiendrait aussi leur ensemble agrégé et cette série quasi-analytique.

Il n'existe donc pas de classe quasi-analytique qui comprenne toutes les autres.

Ce résultat est démontré dans le livre de M. Carleman (page 3) pour les classes que M. Mandelbrojt appelle quasi-analytiques I; mais les deux définitions I et $d$ qui sont équivalentes quand elles s'appliquent à une classe $C_{A_{m}}$ (M. Carleman, loc. cit. page 9) cessent de l'être, même lorsqu'elles se limitent à des fonctions dérivables, pour les classes qui ne sont pas caractérisées par des limitations du type (I), ainsi que l'a démontré M. Mandelbrojt (Séries de Fourier et classes quasi-analytiques, Paris, I935, p. 132).

Séminaire Mathématique de l'Université de Madrid 20-1-42.

R. San Juan.

${ }^{2}$ On sait que Mandelbrojt appelle classe quasi-analytique $A$ une classe de fonctions indéfiniment dérivables dans un intervalle $(a, b)$ lorsque deux fonctions de la classe coïncident dans tout l'intervalle si elles ont des dérivées égales en un point (MANDelbrojt: Séries de Fourier et elasses quasi-analytiques, Paris 1935, page I30); et il appelle quasi-analytique I une classe de fonctions quelconque définies dans un intervalle, lorsque deux fonctions de la classe eoïncident dans tout l'intervalle en tant qu'elles coïncident dans un intervalle partiel $(\alpha, \beta)$ si petit qu'il soit (page 5o). 